\title{
Stillvorbereitung der Eltern
}

\author{
Tatjana Nicin
}

\begin{abstract}
Mit einer ausführlichen und frühen Information schwangerer Frauen und ihrer Familien über die Bedeutung des Stillens tragen Hebammen entscheidend dazu bei, dass Säuglinge nach der Geburt und in den ersten Lebensjahren optimal versorgt werden. Hebamme, Still- und Laktationsberaterin Tatjana Nicin engagiert sich seit Jahren für eine bessere Elternaufklärung und mehr gesellschaftliche Akzeptanz des Stillens. Für DIE HEBAMME reflektiert sie aus Ihrer klinischen und außerklinischen Erfahrung heraus mögliche Gründe für niedrige Stillraten in Deutschland und gibt konkrete Tipps, um Eltern zum Stillen zu motivieren.
\end{abstract}

\section{Die Stillrate - Spiegel der Gesellschaft}

Wie lange Menschen stillen und ob sie überhaupt stillen, ist auch ein Abbild der Gesellschaft, in der sie leben. Darüber müssen wir uns bewusst sein, wenn wir Frauen und Familien beraten. In der Hebammensprechstunde stelle ich Eltern immer die Fragen: „Was meinen Sie, ist normal? Wie lange geben die Menschen ihren Babys Muttermilch?" Eltern mit einer Herkunft aus Ländern mit guten Stillraten sagen alle, es sei normal, „mehrere Jahre“ oder „mindestens ein Jahr“ zu stillen. Eltern, die hierzulande aufgewachsen sind und keinen Stillvorbereitungskurs besucht haben, antworten meist: „4 bis 6 Monate“. Das entspricht auch den bisher erhobenen Stillraten. Aus meiner Beobachtung gibt es dafür verschiedene Ursachen:

- Mangelnde Vorbilder und mangelnde Unterstützung aus der Kernfamilie. Viele werdende Eltern wurden selbst nur 4 bis 6 Monate gestillt. Und Stillen in der Öffentlichkeit ist in Deutschland keineswegs überall gesellschaftlich akzeptiert und willkommen.

- Mangelnde Sachkenntnis: Schwangere Frauen sind zu wenig über die Bedeutung für die Gesundheit, Entwicklung und Bindung sowie über die Praxis des Stillens informiert.

- Krankenhausroutine: teilweise mangelnde oder nicht kompetente Begleitung und Unterstützung zum Thema Stillen.

- Das Marketing der Hersteller künstlicher Babynahrung vermittelt (z. B. in Arbeitsmaterialien, Broschüren, Kursinformationen, Terminblocks, Proben, preiswerten Fortbildungen, finanzieller Hilfe) unterschwellig die Botschaft, Stillprodukte gehörten zur normalen Säuglingsernährung. Auch Fachleute und Fachgesellschaften sind als Multiplikatoren Zielgruppe dieses Marketings.

Hebammen können in der Schwangerenvorsorge und im Krankenhaus mit kompetenter, nicht von wirtschaftlichen
Interessen beeinflusster Elterninformation und Stillberatung Wissenslücken schließen und zu einer positiven Stillerfahrung führen. Uns sollte es nicht egal sein, wie Babys geboren und ernährt werden.

\section{INFO}

Stillempfehlungen

WHO und UNICEF empfehlen, Säuglinge 6 Monate ausschließlich zu stillen und anschließend während der Einführung der Beikost weiterzustillen bis zum Alter von 2 Jahren oder darüber hinaus.

Die Nationale Stillkommission (NSK) am Bundesinstitut für Risikobewertung (BfR) vertritt folgende Empfehlungen:

- Säuglinge sollten mindestens bis zum Beginn des 5. Monats ausschließlich gestillt werden.

- Auch nach Einführung von Beikost - frühestens mit Beginn des 5. Monats, spätestens mit Beginn des 7. Monats - sollten Säuglinge weiter gestillt werden.

- Ab wann ein Säugling innerhalb dieses Zeitfensters zusätzlich Beikost benötigt, ergibt sich individuell in Abhängigkeit von Gedeihen und Essfähigkeit des Kindes.

- Die Stilldauer insgesamt bestimmen Mutter und Kind.

Der NSK gehören Mitglieder aus medizinischen Berufsverbänden und Organisationen an, die sich für die Förderung des Stillens in Deutschland einsetzen. Diese Empfehlungen hat die Kommission gemeinsam mit den pädiatrischen Fachgesellschaften im Rahmen des Netzwerks „Gesund ins Leben - Netzwerk Junge Familie“ konsentiert. Berücksichtigt wurden dabei ernährungsphysiologische Aspekte, Daten zur Auswirkung des ausschließlichen Stillens bzw. der Beikostfütterung auf Wachstum, Entwicklung und spätere Krankheitsrisiken des Kindes. Demnach 
haben Säuglinge, die 4 bis 6 Monate lang ausschließlich gestillt wurden, ein deutlich geringeres Infektionsrisiko zum Beispiel bei Atemwegsinfekten. Weitere Krankheiten, die bei gestillten Kindern im späteren Leben seltener auftreten können, sind Übergewicht und Diabetes mellitus Typ 2.

2014 führte eine Aktualisierung der von medizinischen Fachgesellschaften erarbeiteten S3-Leitlinie zur Allergieprävention zur Verunsicherung von Eltern und Fachpersonal, da sie von bestehenden Empfehlungen abweicht und volles Stillen in den ersten 4 Monaten sowie die Fütterung von Beikost nach dem 4. Monat empfiehlt. Die NSK veröffentlichte dazu eine Stellungnahme und wies darauf hin, sie halte an ihren o.g. Empfehlungen fest, da es keine wissenschaftlichen Gründe für eine Änderung gebe [1].

\section{Zielgruppen der Stillinformation}

Als Hebamme, als Frau, als Stillexpertin bin ich der Ansicht, dass Frauen schon in der Kindheit eine erste Stillinformation erhalten sollten. Sie sollten durch die eigene Stillzeit geprägt und durch positive Einflüsse durch die Gesellschaft gefestigt werden. Dafür sollten wir als Hebammen sowohl die Frauen und betroffenen Familien informieren als auch die Gesellschaft. Wir sollten immer, auch in unserer Freizeit, über Stillen als normale Form der Ernährung reden. Wir sollten in Kitas gehen und Kindern die Selbstverständlichkeit des Gebärens und des Stillens altersgemäß vermitteln, d. h. auf spielerische Weise, z. B. mit Puppen. In Grundschulen und weiterführenden Schulen sollten wir dann Kinder und Jugendliche über die Bedeutung des Stillens - als die normale und selbstverständliche Säuglingsernährung - aufklären. Wir können auch gut Geschwisterkinder bei Hausbesuchen in die Aufklärung einbinden und aufs Stillen vorbereiten, sodass auch sie die Eltern anspornen und motivieren können.

Zum Zweiten sollten wir als Hebammen die Fachleute informieren, mit denen wir arbeiten. Wir können z. B. in Kooperation mit Apotheken anbieten, Vorträge über das Stillen zu halten. Vielleicht lassen sich Apotheker überzeugen, die Werbung für Muttermilchersatznahrung, Fläschchen und Schnuller nicht im Schaufenster zu platzieren. Auch mit Frauen- und Kinderärzten können wir über werbliche Botschaften in Marketingmaterial sprechen und uns gemeinsame Ziele setzen, wie wir Eltern neutral über das Stillen aufklären sollten. Man kann z.B. gemeinsam erarbeiten, welche Stillinformation wir Eltern schriftlich an die Hand geben und wer das persönliche Gespräch übernimmt, das eine solche schriftliche Information immer ergänzen sollte. Wichtig ist, dass wir als Fachleute eine Sprache sprechen und Eltern keine widersprüchlichen Empfehlungen geben. Kontraproduktiv wäre z. B. wenn die Hebamme rät, so lange zu stillen, wie es Mutter und Kind mögen, während der Kinderarzt das Abstillen empfiehlt oder fragt, warum sie im 5. Monat noch keine Beikost gibt. Auf diese Weise verwirren wir als Fachpersonen die Eltern, die sich als Ratsuchende an uns wenden.

Ich persönlich habe die Fachpersonen in meinem Ortsteil nach und nach besucht und mit ihnen besprochen, was wir zu bestimmten Themen den Eltern vermitteln: „Was sagen Sie z. B. zu Geburtsposition oder Stillen, Ernährung oder Medikamenten in der Schwangerschaft und Stillzeit?“ Bei Hausärzten habe ich auch gefragt, welche Literatur sie zu Rate ziehen für ihre Verordnungen. Wir können hier Embryotox empfehlen [2]. Indem man darüber redet, kann man sich auch interprofessionell ergänzen. Ich sage den Ärzten auch, dass die Frauen mich zur Stillberatung anrufen können. So wussten sie, dass ich sie als Hebamme bei der Begleitung von schwangeren Frauen unterstütze.

\section{Den richtigen Ton Treffen}

Bei der Kommunikation der empfohlenen Stilldauer müssen wir darauf achten, dass sich nicht die falsche Botschaft einprägt: Ich hatte schon Eltern in der Hebammensprechstunde, die aus dem Geburtsvorbereitungskurs mitgenommen haben, sie sollten nach 4 Monaten abstillen - so hat das die Hebamme sicher nicht gesagt. Wie aber lässt sich der richtige Ton treffen? Wenn wir Eltern sagen, dass sie ihr Kind in den ersten 6 Monaten ausschließlich stillen sollen, muss die Betonung auf „ausschließlich“ liegen. Dann sagen wir ihnen, dass ihr Baby irgendwann zwischen dem 5. und 9. Monat körperlich so weit ist, dass es auch andere Nahrungsmittel annehmen und verdauen kann. Wenn wir das so vermitteln, klingt es ganz anders und bei den Eltern bleibt tatsächlich hängen, dass sie ihr Kind mindestens ein Jahr stillen sollten.

Wichtig für Hebammen: Beziehen Sie immer auch die Partner der Frauen mit ein. Es gibt Studien, die darauf hinweisen, dass Säuglinge länger Muttermilch bekommen, wenn auch der Vater über die Bedeutung und Vorteile des Stillens aufgeklärt ist. Auch die Geschwisterkinder sollten mit involviert werden. Um zudem die Großeltern ins Boot zu holen, gebe ich den Eltern einen Brief aus Sicht des Babys an die Hand: Diesen können sie mit dem Namen des Babys unterschreiben und darin erklärt quasi das Kind selbst, warum die Eltern es im Tragetuch tragen, warum es lange gestillt wird, warum es Ruhe braucht usw. Das empfinden viele Eltern als sehr hilfreich, um zu vermitteln, was Eltern heute in den ersten Lebensmonaten des Säuglings anders machen als ihre Eltern bei ihnen. Wenn eine Schwangere sagt, dass ihre Mutter großen Einfluss auf ihre Entscheidungen oder ihr Verhalten hat, können wir ihr auch eine Schwangerenaufklärung für Großeltern oder Angehörige empfehlen, damit sie von Anfang über die Bedeutung und Vorteile des Stillens aufgeklärt ist. 
Merke

Was wir den Eltern unbedingt vermitteln müssen: Beikosteinführung bedeutet nicht Abstillen. Wir sollten den Eltern erzählen, dass Stillen über mehrere Jahre normal ist und dass sie ihr Kind mindestens ein Jahr lang stillen sollten, auch nachdem sie die Beikost eingeführt haben.

\section{Die Haltung der Hebamme}

Stillen betrifft jeden von uns. Und Stillen ist ein hoch emotionales Thema. Jeder ist selbst gestillt worden oder eben nicht. Das gilt auch für Hebammen. Somit müssen natürlich auch wir uns fragen, was unsere Stillerfahrung für unser Selbstwertgefühl in der Beratung bedeutet. Es ist wichtig, professionelle Distanz zu wahren: Wenn eine Hebamme selbst nicht gestillt worden ist oder ihre Kinder nicht gestillt hat, bedeutet das nicht, dass sie nicht kompetent zum Stillen beraten kann. Oft fragen mich die Frauen: „Wie lange haben Sie denn gestillt?“ Dann sage ich: „Es geht jetzt nicht um mich. Es geht darum, was gut für das Baby ist." So lässt sich etwa eine Diskussion vermeiden, falls ich selbst nicht so lange gestillt habe. Und falls das doch zur Sprache kommt und ich die Frau motivieren möchte, kann ich ihr sagen: „Ja, das war so, aber ich hatte auch nicht so eine gute Beratung durch meinen Arzt oder meine Hebamme.“

\section{CHECKLISTE}

\section{Formen der Stillinformation}

Nutzen Sie bereits alle Möglicheiten, als Hebamme Stillinformationen weiterzugeben? Mit wem können Sie sich vernetzen? Checken Sie folgende Optionen:

- Stillvorbereitungskurs

- Stillinformationsabend

- Schwangerenvorsorge (gezielt eine Sitzung über das Stillen)

- Geburtsvorbereitungskurs (Empfehlung: 4 Stunden)

- Stillcafé

- Netzwerke mit Stillberatungsstellen für die Weiterempfehlung der Frau (z. B. Hebamme zeigt und erklärt Umgang mit dem Stillkissen - im Stillcafé erhält die Frau vertiefende Informationen; Hebamme bietet selbst keine Geburtsvorbereitungskurse an - empfiehlt dafür Klinik oder Hebammenpraxis in der Nähe)

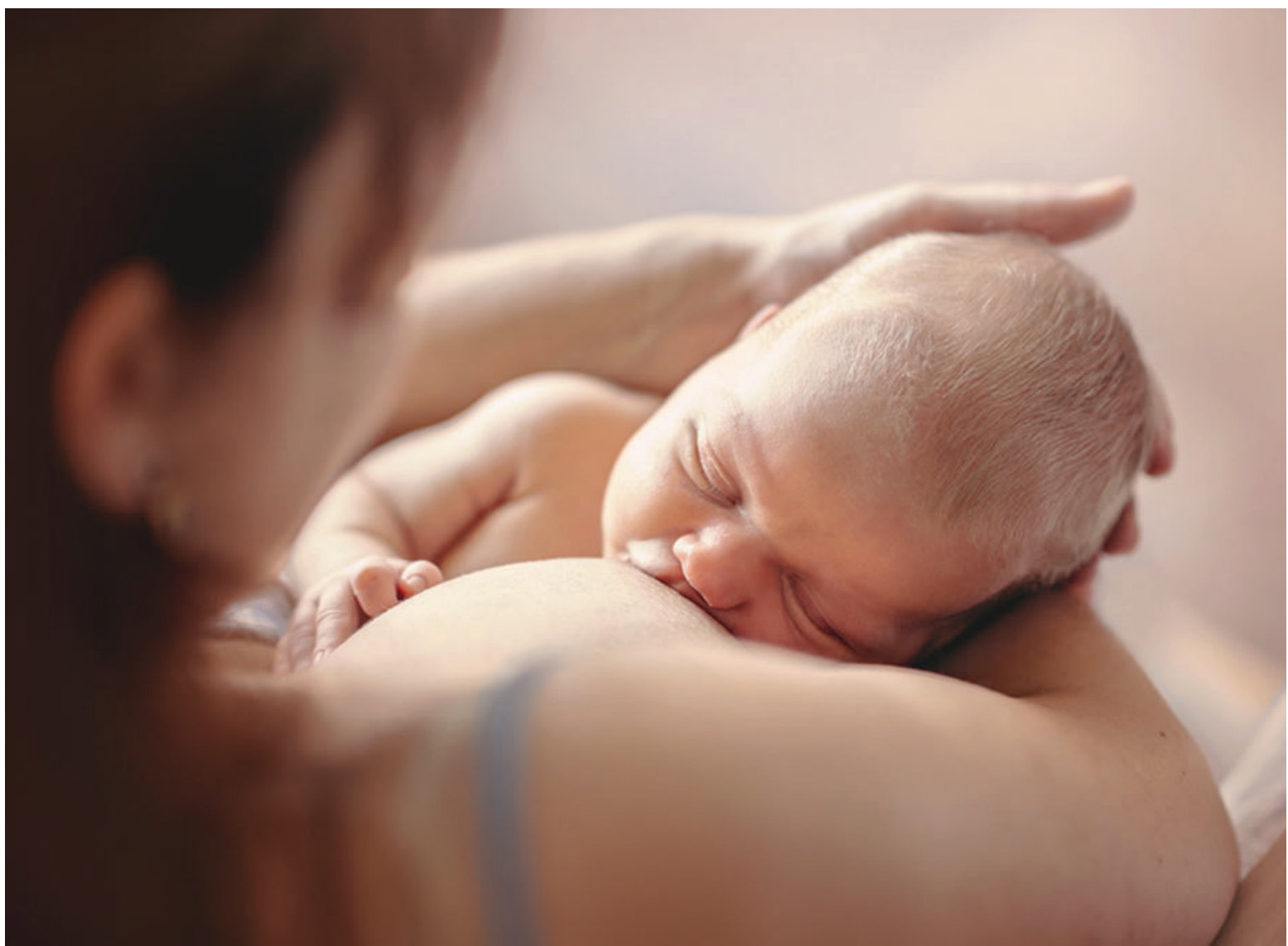

Abb. 1 Mit einer frühen und kompetenten Stillinformation, die Frauen in ihrer Intuition und ihrem Selbstwertgefühl bestärkt, können Hebammen die Basis für eine erfolgreiche und gute Stillbeziehung schaffen. (Quelle: Romanova Anna - stock.adobe.com, Symbolfoto) 


\section{Frühzeitig über das Stillen informieren}

Je früher in der Schwangerschaft wir mit der Frau bzw. den Eltern über das Thema Stillen sprechen, desto besser. Denn es werden schon früh die Abläufe mit dem Baby geplant, z. B. wann die Frau wieder arbeiten gehen wird, welche Babyausstattung sie braucht usw. Lawrence und Lawrence betonen: Erwiesenermaßen führt die Thematisierung der Säuglingsernährung in der frühen Schwangerschaft zu einer Verbesserung von Stillrate und Stilldauer [3].

Hebammen in der Schwangerenvorsorge können Stillinformation ausführlicher vermitteln als Hebammen in der Klinik, wo wir die Schwangere nur sehen, wenn eine Pathologie auftritt oder die Frau zum Geburtsvorgespräch kommt. Zu diesem Zeitpunkt sind die Frauen schon in der 34. bis 36. SSW. Da sprechen wir hauptsächlich noch über Stillzeichen, über das Vermeiden der Trennung von Mutter und Kind und darüber, wie oft das Baby an die Brust kommt. Viele wissen zu diesem fortgeschrittenen Zeitpunkt darüber noch nicht Bescheid.

Aber Stillen hat auch viel mit Bindung zu tun. Und wenn wir früh mit der Frau über Bonding und Bindung sprechen, gewinnt sie automatisch eine positive Haltung gegenüber dem Stillen. Deshalb sollten wir in der Frühschwangerschaft erst mal über Bindung und Bedürfnisse des Babys reden: Schlafen im 1. Lebensjahr, im Elternzimmer, Co-Sleeping, Bed-Sharing - bei all diesen Themen kann Stillen mit einfließen.

Ich plädiere dafür, in der Schwangerenvorsorge nicht nur nach Pathologie oder dem Ausschluss von Pathologie zu schauen. Wenn eine Frau immer nur auf den Mutterpass schaut und jeden Besuch beim Frauenarzt abhakt wie eine bestandene Prüfung, ist sie für die Zeit nach der Geburt noch gar nicht vorbereitet, denn dafür gibt es keinen Mutterpass. Daher sollte die Vorsorge vielmehr auf Aufklärung und Information verlagert werden. Hebammen können das sehr gut: Wir können das Selbstwertgefühl der Schwangeren stärken, ihre Intuition stärken, sie ermutigen zu gebären, Mutter zu sein und zu stillen. Wenn wir Frauen selbstbewusst und stark machen, kommen sie so zur Geburt, trauen sich diese zu und haben danach mit dem Stillen weniger Probleme. Sie erwarten von uns, dass wir ihnen sagen, was sie bei der Geburt und danach machen und wie sie stillen sollen. Es geht darum, dass wir diese Frauen begleiten, unterstützen, ihnen helfen.

\section{Merke}

Die Beratung zur Bedeutung und Praxis des Stillens sollte durch alle, die die Schwangere betreuen, erfolgen. Den werdenden Eltern ist objektives Informationsmaterial (z. B. die Stillinformation der Nationalen Stillkommission im Mutterpass) zu geben, das den aktuellen wissenschaftlichen Erkenntnissen zu Laktation und Stillen entspricht. Die schriftlichen Informationen sollten unbedingt in einem persönlichen Gespräch ergänzt werden.

\section{Ziele der Stillinformation}

Mit dem Ziel, die Stillquoten zu erhöhen muss unsere Stillinformation:

- motivierend wirken

- Umdenkungsprozesse anregen

- Kompetent und neutral informieren

- den Eltern neben Basisinformationen auch vermitteln, wie sie selbstbewusst antworten, z. B. auf Fragen wie: „Was, du gibst keinen Schnuller?“ - „Was, du stillst immer noch...?"

- realistische Erwartungen ansprechen

- Ärzte, Kitas, Schulen ... einbinden (oft fehlen Informationen, Tipps, hilfreiche Adressen. ..)

- für mehr Akzeptanz des Stillens in der Öffentlichkeit werben

\section{INHALTE DER STILLVORBEREITUNG}

- Empfehlungen für das Stillen

- Vorteile des Stillens und der Stilldauer

- Wie lange und wie oft stillen?

- Bedürfnisse des Kindes

- Bedeutung des Wochenbetts

- Was ist Muttermilch (Biochemie)?

- Metabolische Prägung

- Die Gründe für die niedrigen Stillraten in Deutschland, Hindernisse für erfolgreiches Stillen (Kodex, Werbung, mangelnde Fachliche Kompetenzen des medizinisches Personals, Krankenhausroutine ...)

- Ernährung in der Stillzeit

- Brustpflege

- Wann und wie beginnt die Stillbeziehung? (Pränatales Bonding, Geburt, erste Lebensstunden, Hormonstatus des Kindes und der Mutter, Kolostrum)

- Milchtransfer - wie gelangt die Milch von der Mutter zum Baby?

- Stillzeichen

- Anatomie der Brust, Körpergefühl, Selbstvertrauen der Frau

- Milchproduktion (Angebot und Nachfrage)

- Gedeihen des Babys

- Saugvorgang

- Saugverwirrung

- Stillpositionen (intuitives Stillen)

- Schnuller, Beruhigungssauger

- Bonding, Bindung, Beziehung (Rooming-in, Rebonding)

- Schlafen

- Tragen

- Besondere Stillsituationen (Zwillinge, Mehrlinge, Diabetes, Frühgeborene, Sectio, Tandemstillen, 
Stillen und Arbeiten, Medikamente in der Stillzeit, Indikationen und Methoden für Zufütterung, Stillen in der Öffentlichkeit)

- Stillmanagement

\section{Inhalte der Stillinformation - Beispiele aus der Praxis}

Unsere Leitfrage sollte immer lauten: Wo stehen die Frauen bzw. Eltern heute und welche Informationen brauchen sie. Dort müssen wir sie abholen.

\section{Grundsätzliche Bedenken der Frauen ausräumen}

Wenn eine Frau z. B. Bedenken hat, ob es mit dem Stillen klappt, dann sage ich ihr: „Stell dir vor, hier ist ein Berg und oben steht ein wunderschöner Apfelbaum mit phantastischen, leckeren Äpfeln. Ich sage mir, ich gehe hoch und hole mir den Apfel. Und du sagst mir, wenn es klappt, geh ich hoch und hole mir den Apfel. Wer von uns beiden wird sich den Apfel wohl holen?" Dann versteht die Frau sofort, dass sie es sich mit dieser Einstellung schwerer macht mit dem Stillen. Dass erst mal der
Wille da sein muss, um Kraft dafür zu haben. Sie kann sich sagen: „Ich werde das tun. Und um das zu tun, muss ich dieses und jenes wissen. Und das mit meinem Mann, mit meiner Familie, mit meinen Freunden klären - und dann klappt es. Es wird klappen, ich will das. Und selbst wenn es Probleme gibt, dann habe ich meine Hebamme oder meine Stillambulanz, meine Stillberaterin oder eine Frauenärztin, die mich zum Thema Stillen beraten und unterstützen.“ Es muss also erst einmal diese Grundhaltung geschaffen werden und dann können wir Inhalte vertiefen. Dafür eignen sich Stillvorbereitungskurse oder Stillinformationsabende. Wir bieten in der Stillambulanz unserer Klinik auch eine Stillberatung an.

\section{Besondere Stillsituationen}

Manche Frauen sind unsicher, ob sie stillen können, weil sie sich die Brust haben operieren lassen. Dann untersuche ich die Brust. Meist kann ich die Frau beruhigen: Erst mal sollte sie mit dem Stillen starten und schauen, ob überhaupt Probleme auftreten. Ich sage ihr dann, sie soll stillen, davon ausgehen, dass alles gut gehen wird, und dass ich das beobachte. Dadurch kann sie sich ganz auf ihr Selbstbewusstsein konzentrieren und hat zugleich die Sicherheit, dass jemand auf sie aufpasst. 
Wenn eine Frau Hohlwarzen hat, kann man schon in der Schwangerschaft, mit Brustwarzenformer arbeiten allerdings nur wenn die Frau es möchte. Hilfreich ist es auch, ihr Tipps fürs Anlegen zu geben oder ihr auf der Wochenbettstation Stillpositionen zu zeigen (z.B. Rückengriff, C-Griff). Praktische Tipps helfen, Stillprobleme in den Griff zu bekommen. Und natürlich können wir auch die Frau beruhigen und sagen: „Es gibt viele Frauen, die trotzdem gut gestillt haben. “ Also, sie beruhigen und motivieren.

Wenn eine Frau krank ist und Medikamente einnehmen muss, kann man mit Embryotox telefonieren. Das Pharmakovigilanz- und Beratungszentrum für Embryonaltoxikologie der Charité-Universitätsmedizin Berlin berät unabhängig zum Risiko von Arzneimitteln, die während der Schwangerschaft und der Stillzeit zum Einsatz kommen. So lässt sich prüfen, ob man Medikamente ändern könnte oder was die Frau braucht, um doch stillen zu können. Und oft kann sie dann stillen. In Ausnahmefällen dürfen Frauen tatsächlich nicht stillen, aber das können wir eben abklären.

Manche Frauen fühlen sich beruflich unter Druck und glauben, dass sie nicht stillen können, weil sie nach ein paar Wochen schon wieder arbeiten gehen müssen. Dann kläre ich sie über ihre Rechte auf und bestärke sie darin, mit ihrem Arbeitgeber darüber zu sprechen. So lässt sich meist eine gute Lösung finden. Viele kennen das Mutterschutzgesetz nicht, in dem Stillpausen am Arbeitsplatz geregelt sind (während der ersten 12 Monate nach der Geburt: mindestens zweimal täglich eine halbe Stunde oder einmal pro Tag eine Stunde). Zudem verbietet es Arbeitgebern, stillende Frauen Arbeitsbedingungen auszusetzen, bei denen eine unverantwortbare Gefährdung ihrer physischen oder psychischen Gesundheit besteht. Eine laienverständliche Broschüre mit allen Informationen steht auf der Website des Bundesministeriums für Familie, Senioren, Frauen und Jugend zum Download bereit [4]. Der Hausarzt, Frauenarzt oder Betriebsarzt kann ein entsprechendes Beschäftigungsverbot in der Stillzeit aussprechen.

Frauen mit Diabetes oder Schwangerschaftsdiabetes haben manchmal einen geringeren Milcheinschuss oder eine geringere Muttermilchmenge. Ich motiviere diese Frauen, ihre Muttermilch für die Zeit unmittelbar nach der Geburt zu sammeln. Laut Leitlinie soll das Kind der diabetischen Mutter in der ersten halben Stunde postpartum Nahrung (hoffentlich Muttermilch, sonst Muttermilchersatznahrung) erhalten und nach 1 Stunde muss man den ersten Blutzuckerwert messen. Wir wissen, dass viele Babys erschöpft sind nach der Geburt, weil diabetische Schwangerschaften oft eingeleitet werden. Dann gehen diese Kinder nicht in der ersten halben Stunde an die Brust. Die Frauen sind je nach Geburtsmodus und -verlauf manchmal ebenfalls erschöpft. Wenn das Kind der diabetischen Mutter in der ersten halben Stunde Muttermilch bekommt, sind die Blutzuckerwerte viel besser, der Säugling ist stabiler und neigt weniger dazu, später Diabetes zu bekommen. Daher zeige ich den Frauen in der Hebammensprechstunde, wie sie mit der Hand Kolostrum gewinnen können. Sie können 72 Stunden sammeln, die Muttermilch im Kühlschrank aufbewahren und tiefgefrieren, mit ununterbrochener Kühlkette, in die Klinik mitbringen. Meine Erfahrung ist, dass es dadurch den Babys viel besser geht und die Laktation nach der Geburt schneller einsetzt.

Es gibt Frauen, die ambivalent sind, weil der Partner gegen das Stillen ist. Ich frage dann die Frau, ob ich mit ihrem Mann darüber reden soll. Dann schlage ich vor, dass wir noch einen Termin mit ihm ausmachen, weil ich ihm sowieso das Tragen mit dem Tragetuch beibringen oder mit ihm übers Wochenbett sprechen wollte. Wenn die beiden keinen Vorbereitungskurs gemacht haben, vereinbare ich grundsätzlich einen Termin, bei dem der Partner dabei ist, um mit beiden über Wochenbett, Stillen, Tragen, Bedürfnisse des Kindes, Organisation, Unterstützungsmöglichkeiten des Vaters usw. zu sprechen. Dabei können wir dann auch Tabus offen diskutieren, z. B.: Was führt dazu, dass manche Männer das Stillen in der Öffentlichkeit ablehnen? Ist die Brust in ihren Augen ein Nährorgan oder ein Sexorgan? Man kann mit dem Paar über das Thema Sexualität in der Stillzeit sprechen. Meist hilft auch hier Aufklärung.

\section{Merke}

Bei besonderen Stillsituationen ist immer eine gute Beratung wichtig, das gilt insbesondere bei Mehrlingen, drohender Frühgeburt, geplanter Sectio, Tandemstillen, Diabetes, wenn die Frauen zeitig nach der Geburt wieder arbeiten gehen, wenn sie Medikamente einnehmen, wenn Babys zugefüttert werden müssen.

\section{Stillvorbereitungskurse - Tipps für die Praxis}

Ich arbeite in meinen Stillvorbereitungskursen viel praktisch mit den Eltern. Einige Anregungen dazu:

\section{Zeitkuchen malen}

Ich lasse Eltern jeweils zwei „Zeitkuchen“ ausmalen - einen vor der Geburt und einen nach der Geburt. Dafür bitte ich sie, auf einem Blatt Papier die eingezeichneten Kreise tortenstückförmig mit Alltagstätigkeiten zu füllen: Welchen Anteil haben schlafen, arbeiten, Partnerschaft, Haushalt, Kinder, Hobbys, Körperpflege, Freunde, Familie, Zeit zur freien Verfügung, Baby). Ein Kreis (= 24 Stunden) stellt dabei einen Durchschnittstag dar. Dadurch wird vorstellbar, wie sich die Bedürfnisse des Babys auf den Tagesablauf auswirken. 
Im ersten Moment kann das Angst machen. Die Eltern sollen dann in eine Tabelle eintragen, wer nach der Geburt welche Aufgabe übernimmt (Frühstück machen, Hauptmahlzeit zubereiten, einkaufen gehen, Wohnung aufräumen, Wohnung sauber halten, Wäsche waschen, aufhängen, bügeln, Baby wickeln, baden, tragen...). So wird alles besser einschätzbar und die Eltern sehen, dass sich die Aufgaben gemeinsam - vielleicht mithilfe der Großeltern o. a. - meistern lassen.

Anhand der Zeitkuchen und Aufgabentabelle lässt sich erklären, wie wichtig es ist, dem Kind in dieser Phase mit Empathie entgegenzukommen und wie gering der Aufwand in unserem Leben im Vergleich zu dem großen Gewinn ist, den wir dem Kind als Basis dadurch mitgeben. Das motiviert Eltern sehr. Zudem ist die Tabelle eine gute Basis, um vor der Geburt die Aufgabenverteilung mit den Großeltern und ggf. weiteren Helfern zu besprechen. Das gibt den Eltern Sicherheit und erleichtert ihnen manches, wenn das Baby da ist. Den Vater können wir bei dieser Gelegenheit auch darauf vorbereiten, dass die Tage nicht mehr so planbar sind: Dass er z. B. seinen wöchentlichen Fußballabend machen kann, wenn alles gut läuft, dass er aber darauf verzichten sollte, wenn die Frau wunde Brustwarzen hat und dann weinend allein zu Hause sitzt.

\section{Assoziationen austauschen}

In dieser Übung geht es um die Bedürfnisse des Babys und welche davon den Eltern einfallen. Die Eltern erhalten dafür ein Papier mit senkrecht in Großbuchstaben geschrieben Worten, z. B. S-T-I-L-L-E-N, und sie sollen gemeinsam je ein Schlagwort zu den Buchstaben finden, das ein Bedürfnis ausdrückt (Was braucht mein Baby?) Oft fallen den Eltern erst mal materielle Dinge ein, die Ausstattung fürs Kinderzimmer, Babysachen, die noch einzukaufen sind. Ich sage ihnen dann, dass noch ganz andere Dinge wichtig sind, z. B. Bonding, Zeit haben fürs Baby, Gelassenheit, Liebe, dass sie entspannt bleiben, sich selbst und das Baby gut ernähren. Also ich setze eher auf die Skills der Eltern als auf die Hilfsmittel.

\section{Zettel sortieren}

Für diese Übung liegen eine Reihe kleiner laminierter Kärtchen auf dem Tisch, auf denen bestimmte Dinge stehen z. B. Schnuller, Flasche, Liebe, Nähe, Tragetuch, Autositz, Mobile, Körperkontakt, Gelassenheit, Teetasse, Handy ... Die Eltern sollen diese Zettel in 2 Körbe sortieren, auf dem einen steht „nützlich“ auf dem anderen „nicht nützlich“. Darauf aufbauend entstehen sehr gute Gespräche, von denen die Eltern ganz viel mitnehmen können. Ich kann ihnen dann sagen, was aus meinem Wissen und meiner Erfahrung heraus wichtig ist und was ein Problem werden könnte.

\section{Quiz spielen}

Je nachdem, ob sich die Eltern darauf einlassen, lässt sich auch ein Quiz mit ihnen machen: Wie viel nimmt mein 
Baby in den ersten Tagen zu? Wann verdoppelt sich das Gewicht? Oder die Körperlänge? Dabei kann man auch viel Wissen über das Stillen und konkrete Tipps dazu spielerisch vermitteln.

\section{Stillen üben}

Um den Unterschied zwischen Stillen und Fläschchenfütterung zu veranschaulichen, simuliere ich die Brust mit Luftballons, die mit Wasser gefüllt sind. Die Teilnehmer des Stillvorbereitungskurses saugen an dem Ballon und dann an der Flasche und beschreiben den Unterschied. Das ist für mich ein guter Einstieg, um mit ihnen z. B. über Saugmuster oder Saugverwirrung zu sprechen.

\section{Merke}

Grundregel für den Stillvorbereitungskurs: Alle Teilnehmer gehen würdevoll und respektvoll miteinander um. Jeder darf sagen, was er denkt. Wir arbeiten nicht dogmatisch. Wir arbeiten ohne Druck. Die Teilnehmer erlernen und erleben die Bedeutung und Vorteile des Stillens, werden jedoch nicht verängstigt für den Fall, dass sie z. B. aufgrund geringer Laktation doch auf Muttermilchersatznahrung zurückgreifen müssen.

FAZIT

\section{Elternaufklärung rund ums Stillen}

Still-Informationen sollte eine Frau schon während der Kindheit bekommen.

Förderlich für erfolgreiches Stillen ist es, wenn Frauen selbst gestillt worden sind und in der Kindheit Stillen als etwas Normales/Selbstverständliches vermittelt bekommen haben. Frauen sollten durch positive Einflüsse in der Gesellschaft in ihrer positiven Haltung gegenüber dem Stillen bestärkt werden. Fachleute sollten diesen Prozess begleiten - in Kindergarten, Schule, Medien.

Frauen sollten in ihrer Intuition bestärkt werden, auch dazu, ihre Weiblichkeit zu bewundern und erste Brustspannungen in der Schwangerschaft zu genießen.

Die Selbstverständlichkeit des Gebärens und des Stillens sollte unterstützt werden.

Durch die ganze Schwangerschaft hindurch sollte kontinuierlich über die Bindung und das Stillen informiert werden. Der Schwerpunkt muss dabei weggehen von der Suche nach Pathologie und sich in Richtung Aufklärung, Beobachtung und Verstärkung der Physiologie verlagern.

\section{Autorinnen / Autoren}

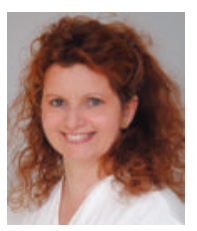

Tatjana Nicin ist Hebamme, Still- und Laktationsberaterin IBCLC, und Sonderpädagogin. Sie arbeitet als pflegerische Bereichsleiterin für Kreißsaal, Elternschule und Mutter-KindStation am Klinikum Hanau.

\section{Korrespondenzadresse}

E-Mail: tnicin@googlemail.com

Literatur

[1] https://www.bfr.bund.de/cm/343/update-der-s3-leitlinie-alle rgiepraevention-weicht-von-stillempfehlung-der-nationalen-s tillkommission-ab.pdf

[2] www.embryotox.de

[3] Lawrence RA, Lawrence RM. Breastfeeding. 8th Edition. A Guide for the Medical Profession. Elsevier; 2015

[4] https://www.bmfsfj.de/blob/94398/ff6a51cf550dbb2c4b6170 cce99e5b3d/mutterschutzgesetz-data.pdf 